\title{
ERRATUM
}

Open Access

\section{Erratum to: Optimal surveillance network design: a value of information model}

\author{
Matteo Convertino ${ }^{1,2,3,4^{*}}$, Yang Liu ${ }^{1}$ and Haejin Hwang ${ }^{1}$
}

\author{
${ }^{*}$ Correspondence: \\ matteoc@umn.edu \\ 1 School of Public Health, \\ Environmental Health \\ Sciences Division and Public \\ Health Informatics Program, \\ HumNat Lab, University \\ of Minnesota, Minneapolis, \\ MN, USA \\ Full list of author information \\ is available at the end of the \\ article
}

\section{Erratum to: Complex Adaptive Systems Modeling 2014, 2:6 DOI 10.1186/s40294-014-0006-8}

The plot of another review paper in progress about diffusion processes on networks was accidentally provided. The correct plot (Additional file 1: Figure S3b) is provided.

\section{Additional file}

Additional file 1: Figure S3b. Outbreak waves and wave velocity error. The top plot shows the error in the outbreak velocity as a function of the value of information related to different surveillance network topologies for the outbreaks considered in this paper. The error is related to the misreporting of cases in space and time. The bottom plot is showing outbreak waves related to the epidemic considered; from left to right epidemic waves are shown for different instantiations from the beginning to the end of the epidemic spanning geographical distances ( $x$-axis). $j_{n}$ is the normalized number of infected dependent on time and distance. The outbreak source is at distance equal to zero.

\section{Author details}

'School of Public Health, Environmental Health Sciences Division and Public Health Informatics Program, HumNat Lab, University of Minnesota, Minneapolis, MN, USA. ${ }^{2}$ Institute on the Environment, University of Minnesota, Minneapolis, MN, USA. ${ }^{3}$ Institute for Engineering in Medicine, University of Minnesota, Minneapolis, MN, USA. ${ }^{4}$ Academic Health Center, Mayo Memorial Building, Suite 1232 12th fl., MMC807, 420 Delaware St. SE, Minneapolis, MN 55455, USA.

The online version of the original article can be found under doi:10.1186/s40294-014-0006-8.

Received: 8 June 2016 Accepted: 9 June 2016

Published online: 22 June 2016 